\title{
Rat BodyMap transcriptomes reveal unique circular RNA features across tissue types and developmental stages
}

\author{
TONG ZHOU, ${ }^{1,5}$ XUEYING XIE, ${ }^{2,5}$ MUSHENG LI, ${ }^{2,5}$ JUNCHAO SHI, ${ }^{1}$ JIN J. ZHOU, ${ }^{3}$ KENNETH S. KNOX, \\ TING WANG, ${ }^{4}$ el CHEN, ${ }^{1}$ and WANJUN GU ${ }^{2}$ \\ ${ }^{1}$ Department of Physiology and Cell Biology, The University of Nevada, Reno School of Medicine, Reno, Nevada 89557, USA \\ ${ }^{2}$ State Key Laboratory of Bioelectronics, School of Biological Sciences and Medical Engineering, Southeast University, Nanjing, \\ Jiangsu 210096, China \\ ${ }^{3}$ Department of Epidemiology and Biostatistics, The University of Arizona, Tucson, Arizona 85721, USA \\ ${ }^{4}$ Department of Internal Medicine, College of Medicine Phoenix, The University of Arizona, Phoenix, Arizona 85004, USA
}

\begin{abstract}
Circular RNAs (circRNAs) are a novel class of regulatory RNAs. Here, we present a comprehensive investigation of circRNA expression profiles across 11 tissues and four developmental stages in rats, along with cross-species analyses in humans and mice. Although the expression of circRNAs is positively correlated with that of cognate mRNAs, highly expressed genes tend to splice a larger fraction of circular transcripts. Moreover, circRNAs exhibit higher tissue specificity than cognate mRNAs. Intriguingly, while we observed a monotonic increase of circRNA abundance with age in the rat brain, we further discovered a dynamic, age-dependent pattern of circRNA expression in the testes that is characterized by a dramatic increase with advancing stages of sexual maturity and a decrease with aging. The age-sensitive testicular circRNAs are highly associated with spermatogenesis, independent of cognate mRNA expression. The tissue/age implications of circRNAs suggest that they present unique physiological functions rather than simply occurring as occasional by-products of gene transcription.
\end{abstract}

Keywords: circRNA expression; testis function; tissue specificity; age-dependent expression

\section{INTRODUCTION}

Circular RNAs (circRNAs) are a class of endogenous RNAs with closed loop structures (Jeck and Sharpless 2014). Many studies have revealed abundant circRNAs in organisms across the eukaryotic tree of life (Jeck et al. 2013; Wang et al. 2014). CircRNAs are mainly formed through pre-mRNA back-splicing (Jeck and Sharpless 2014), which can be regulated by factors such as flanking intronic sequences (Zhang et al. 2014), RNA-binding proteins (Conn et al. 2015), canonical RNA splicing signals (Starke et al. 2015), and exon-containing lariat precursors (Barrett et al. 2015). CircRNAs present various biological functions, including acting as miRNA sponges (Memczak et al. 2013), transcriptional regulation of their parental genes (Li et al. 2015b), and RNA splicing regulation of their cognate messenger RNA (mRNA) (Conn et al. 2017). Moreover, several recent studies have provided strong

\footnotetext{
${ }^{5}$ These authors contributed equally to this work.

Corresponding authors: wanjungu@seu.edu.cn, tongz@med. unr.edu

Article is online at http://www.rnajournal.org/cgi/doi/10.1261/rna. 067132.118. Freely available online through the RNA Open Access option.
}

evidence that some circRNAs can be translated in a cap-independent manner (Legnini et al. 2017; Pamudurti et al. 2017; Yang et al. 2017). The expanding view of circRNA biogenesis and function suggests that circRNAs are a novel class of RNAs with important biological implications.

Given the regulatory functions of circRNAs in gene expression, investigation of the dynamic expression of circRNAs across different cell types, tissues, and organisms is helpful for understanding their roles in various biological processes. CircRNAs are conservatively expressed from orthologous genomic regions between humans and mice (Jeck et al. 2013; Guo et al. 2014) and among Drosophila species (Westholm et al. 2014). Detailed analyses of circRNA expression in humans (Guo et al. 2014; Liu et al. 2016; Xia et al. 2016), mice (Xia et al. 2016), pigs (Liang et al. 2017b), and flies (Westholm et al. 2014) have revealed substantial tissue-specific patterns of circRNA expression. Notably, circRNAs are enriched and abundantly expressed in some specific tissue types and blood components, such as the brain (Westholm et al. 2014; Rybak-Wolf

(C) 2018 Zhou et al. This article, published in RNA, is available under a Creative Commons License (Attribution 4.0 International), as described at http://creativecommons.org/licenses/by/4.0/. 
et al. 2015; Szabo et al. 2015; Veno et al. 2015), testes (Liang et al. 2017b), peripheral whole blood (Memczak et al. 2015), peripheral blood mononucleotide cells (Qian et al. 2018), platelets (Alhasan et al. 2016), and exosomes (Li et al. 2015a). CircRNAs have also been related to the development of the fetal human brain (Szabo et al. 2015), mouse brain (You et al. 2015), fetal porcine brain (Veno et al. 2015), and Drosophila neural systems (Westholm et al. 2014). Interestingly, neural expression of some circRNAs in flies has been suggested as a potential biomarker of aging (Westholm et al. 2014). In addition, aberrant circRNA expression is related to human diseases (Chen et al. 2016), including human cancers (Meng et al. 2017), neural degenerative diseases (Kumar et al. 2017), hematological malignancies (Bonizzato et al. 2016), and infectious diseases (Qian et al. 2018).

Although substantial advances have been made in understanding circRNA expression and its potential function, little is known about the correlation between the expression of circRNAs and mRNAs transcribed from the same host genes. Since circRNAs can either promote the transcription of their host gene (Li et al. 2015b) or regulate the splicing of cognate mRNAs (Conn et al. 2017), the correlation between circRNAs and their linear counterparts can be dynamically regulated. Therefore, it is important to obtain an in-depth understanding of the relationship between the expression profiles of circRNAs and their cognate mRNAs across tissue types and developmental stages. Some previous studies (Guo et al. 2014; Liang and Wilusz 2014; Conn et al. 2015; Rybak-Wolf et al. 2015; You et al. 2015; Chen 2016) have shown that there is no clear correlation between the expression values of circRNAs and their corresponding mRNAs. However, these conclusions might be preliminary and premature, since the circRNA profiles examined in these studies were derived from relatively small data sets, such as RNA sequencing (RNA-seq) data from a single tissue type (Conn et al. 2015; Rybak-Wolf et al. 2015; You et al. 2015), the results of mini-gene experiments (Liang and Wilusz 2014), or data from several different tissues and cell types from different publications (Guo et al. 2014). Since a batch effect inevitably exists in high-throughput sequencing data collected from various sources (Leek et al. 2010), it is technically difficult to conduct a comprehensive comparison of mRNA and circRNA expression across tissues or developmental stages. In addition, the computational estimation of expression values for both linear and circular transcripts based on rRNA-depleted RNA-seq data may not be optimized (Gao and Zhao 2018). The first reason for this lack of optimization is that circRNA expression values in previous studies (Guo et al. 2014; Conn et al. 2015; Rybak-Wolf et al. 2015; You et al. 2015) have been quantified based on the ratio of back-splicing reads to canonical linear reads at a given junction from RNA-seq data. However, this count-based quantification method is less accurate than model-based approaches (Kanitz et al. 2015). The second reason is that canonical reads corresponding to circular transcripts could be misassigned with their corresponding linear transcripts using classical RNA-seq quantification tools. Therefore, it is important to consider both circular and linear transcripts when quantifying RNA expression values from RNA-seq data.

To overcome the above issues, we analyzed the transcriptomes of both mRNAs and circRNAs in a rat BodyMap RNAseq data set (Yu et al. 2014a,b) using Sailfish-cir (Li et al. 2017), a computational tool that we recently developed, which applies a model-based algorithm to precisely quantify expression levels of both linear and circular transcripts from rRNA-depleted RNA-seq data. The rat BodyMap data set contains 320 samples isolated from Fischer 344 rats across 11 tissues and four developmental ages ( $\mathrm{Yu}$ et al. $2014 a, b)$. This is an ideal data set for a systematic comparison of expression values of circRNAs and its cognate mRNAs across tissue types and developmental stages. Using the rat BodyMap data set, we compiled a repertoire of circRNAs in the rat transcriptome and summarized the expression profiles of both circRNAs and mRNAs in all these 320 samples. We explored the expression patterns of circRNAs and linear mRNAs across 11 rat tissues and four developmental stages and investigated the relationship in expression between circRNAs and their linear counterparts. Furthermore, we looked into the roles of circRNAs in determining the tissue's phenotypes and their relations to rat development, and we compared their biological implications with those of linear mRNAs. We found that (i) circRNAs are evolutionarily more conserved than mRNAs; (ii) although the expression of circRNAs is positively correlated with that of cognate mRNAs, genes with higher expression levels tend to show a significantly larger fraction of spliced circular transcripts than their linear counterparts; (iii) circRNAs exhibit higher tissue specificity than mRNAs; (iv) circRNA abundance monotonically increases with age in the rat brain, which is consistent with observations made in humans (Szabo et al. 2015), mice (You et al. 2015; Gruner et al. 2016), and flies (Westholm et al. 2014); and (v) testes circRNA expression shows a dynamic age-dependent pattern, with a dramatic increase with advancing stages of sexual maturity $(2,6$, and $21 \mathrm{wk})$, followed by a decrease with aging (104 wk). The age-sensitive testicular circRNAs are highly associated with spermatogenesis (e.g., cilium morphogenesis and spermatid development) and independent of the expression of their cognate mRNAs. Our study elucidates a complex landscape of circRNA expression across different tissues and developmental stages, and a thorough comparison of circRNA expression profiles against their linear counterparts provides us with a deeper understanding of the biological roles of circular transcripts in tissue specificity and development as well as their relationship with linear mRNAs. 


\section{RESULTS}

\section{A comprehensive rat circRNA repertoire}

To analyze the expression profiles of circRNAs in the rat BodyMap data set, a reference library of known rat circRNAs is required (see Materials and Methods for details), but no such library is available in public databases (Glažar et al. 2014; Liu et al. 2016). Therefore, we first constructed a repertoire of rat circRNAs from 320 samples. Because it is difficult to distinguish circRNA isoforms that are generated by the same back-splicing event using short-read RNA-seq data (Gao et al. 2016), all circRNA isoforms with the same back-splicing junction were considered "circRNA species." The subsequent analyses described in this section were performed at the circRNA species level.

Using our computational pipeline, a total of 16,745 circRNA species were identified in the rat BodyMap data set. Among different rat tissues, the brain expressed the greatest number of circRNA species, while the liver expressed the fewest circRNA species (Fig. 1A). The majority $(86.6 \%, 14,493$ out of 16,745$)$ of rat circRNA species were derived from exonic regions (Fig. 1B). In comparison, only $1223(7.3 \%)$ and 1029 (6.1\%) circRNA species originated from intronic and intergenic regions, respectively (Fig. 1B). Among the exonic circRNAs, most were composed of less than five exons, although some contained more than 20 exons (Fig. 1C). As expected, the length of exonic circRNAs was significantly and positively correlated with the number of exons (Spearman's rank correlation test: $P<10^{-10}$; Supplemental Fig. S1), and most rat exonic circRNAs were less than 1000 base pairs in length (Fig. 1D). Given that exonic circRNAs were predominant in the rat circRNA repertoire, we focused on only exonic circRNAs in the rest of this study.

We next analyzed the evolutionary conservation level (i.e., PhastCons score) (Pollard et al. 2010) at the three codon positions in both circRNA and mRNA exons. We found that the conservation level of exonic circRNAs was significantly higher than that of mRNAs (Kolmogorov-Smirnov test: $P<10^{-10}$ for all three codon positions; Fig. 1E). This finding suggests that circRNAs may be under some extra selective pressure to ensure their proper biogenesis and/ or functions, such as binding of splicing factors or RNAbinding proteins. Furthermore, we investigated the evolutionary dynamics of circRNA biogenesis in the mammalian lineage. We compared the rat circRNA repertoire with the human and mouse circRNA repertoires. We observed that most of the rat exonic circRNA species occurred in all three mammalian transcriptomes (Fig. 1F). In comparison, only 997 (6.9\%) of the rat exonic circRNAs evolved recently, after the separation of the common ancestor of rodents and humans (Fig. 1F). Additionally, 861 (5.9\%) of the rat exonic circRNAs evolved specifically in the rat lineage (Fig. 1F). These rat-specific and rodent-specific circRNAs contained
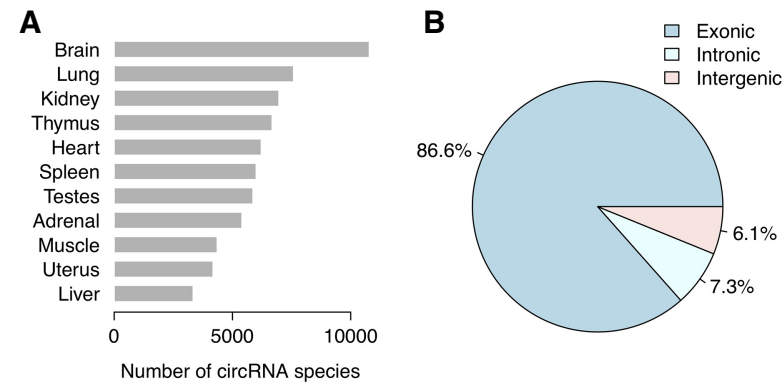

E

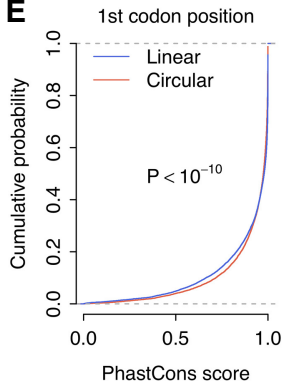

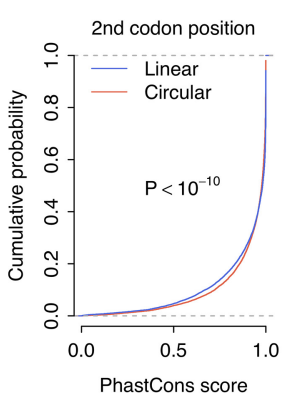
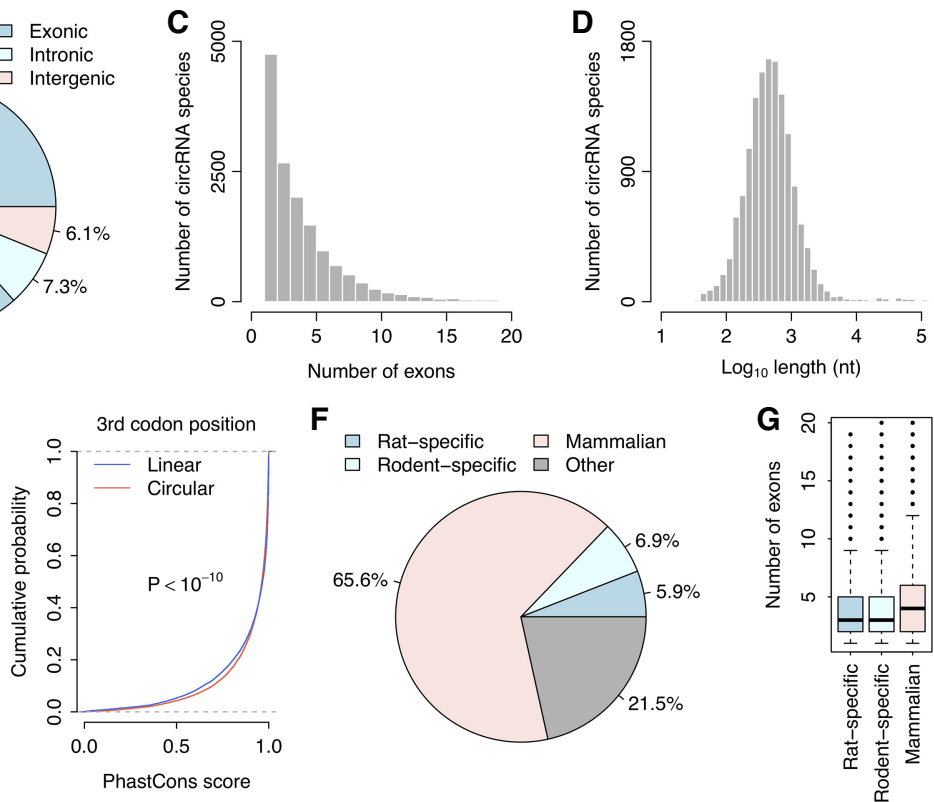

$\mathbf{F}$ $\square$ Rat-specific $\square$ Mammalian $\square$ Rodent-specific $\square$ Othe
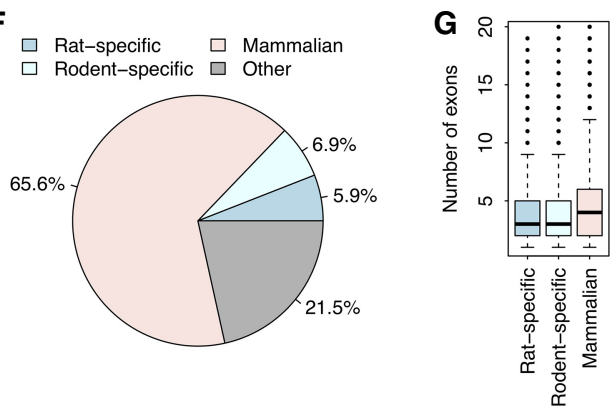

FIGURE 1. The rat circRNA repertoire. (A) Comparison of the number of circRNA species across the 11 rat tissues. (B) Fractions of exonic, intronic, and intergenic circRNA species within the repertoire. (C) Histogram of the number of exons in the exonic circRNAs of rats. (D) Histogram of circRNA length in rats. $(E)$ Cumulative distribution of the PhastCons scores of both linear and circular transcripts at the three codon positions. A significantly increased PhastCons score was observed for the circular transcripts compared with the linear transcripts. The $P$-values were calculated with the Kolmogorov-Smirnov test. (F) Fractions of mammalian-common, rodent-specific, and rat-specific circRNA species within the repertoire. (G) Comparisons of exon numbers among the mammalian-common, rodent-specific, and rat-specific circRNAs. 
fewer exons than the mammalian-common circRNAs (Wilcoxon test: $P<10^{-10}$; Fig. 1G), which suggests that younger circRNAs are more likely to be short in length. Given the importance of flanking RNA structure in circRNA biogenesis (Ashwal-Fluss et al. 2014; Liang and Wilusz 2014; Zhang et al. 2014), it is reasonable to observe relatively shorter circular transcripts in evolutionarily younger circRNA species.

\section{Relationship between the expression of circular and linear transcripts}

Based on the rat circRNA repertoire described above, we estimated the expression levels of both circular and linear transcripts in all rat BodyMap samples using our recently published RNA-seq quantification framework, Sailfish-cir (Li et al. 2017). For each host gene, the transcripts per million (TPM) values of both circular (TPM circ) and linear transcripts $\left(T P M_{\text {linear }}\right)$ were calculated. We observed a significant positive correlation between the expression of circRNAs and their linear counterparts in all 11 tissue types (Spearman's rank correlation test: $P<10^{-10}$; Fig. 2A,B; Supplemental Fig. S2), which suggests that circRNA expression is largely regulated at the transcriptional level of the corresponding host genes. Across the 11 tissue types, the expression of circRNAs was significantly lower than that of their linear counterparts (paired t-test: $P<10^{-10}$ ). However, there was a considerable number of circRNAs with relatively high expression levels, exceeding those of their linear counterparts (Fig. 2A; Supplemental Fig. S2).
For example, TPM $M_{\text {circ }}$ was found to be higher than TPM $M_{\text {linear }}$ for 462 host genes in the rat brain (Fig. 2A). We examined the Gene Ontology Biological Process (GOBP) (Ashburner et al. 2000) terms associated with the host genes of these "special" circRNAs. We found that, across almost all the tissue types, there were several common GOBP terms associated with these circRNAs, such as "protein phosphorylation," "intracellular signal transduction," "positive regulation of GTPase activity," and so on (Supplemental Fig. S3). We also calculated the fraction of circular transcripts [TPM $\left.M_{\text {circ }} /\left(T P M_{\text {circ }}+T P M_{\text {linear }}\right)\right]$ for individual host genes. Interestingly, we found that, across the 11 tissue types, genes with higher expression levels tended to exhibit a significantly larger fraction of spliced circular transcripts than their linear counterparts (Spearman's rank correlation test: $P<10^{-10}$; Fig. 2C), which suggests that circRNAs are more "sensitive" to alterations of host gene expression than mRNAs. This observation also implies that in addition to being subject to transcriptional regulation of host genes, the expression level of circRNAs may also be controlled at the splicing level.

\section{CircRNAs show higher tissue specificity than mRNAs}

Tissue-specific expression has been systematically investigated for mRNAs but not circRNAs (Fagerberg et al. 2014; Yu et al. 2014a; Andergassen et al. 2017). To understand the extent to which circRNA expression shows a tissuedependent pattern, we performed a principal component analysis (PCA) of circRNA expression for all the rat tissue
A
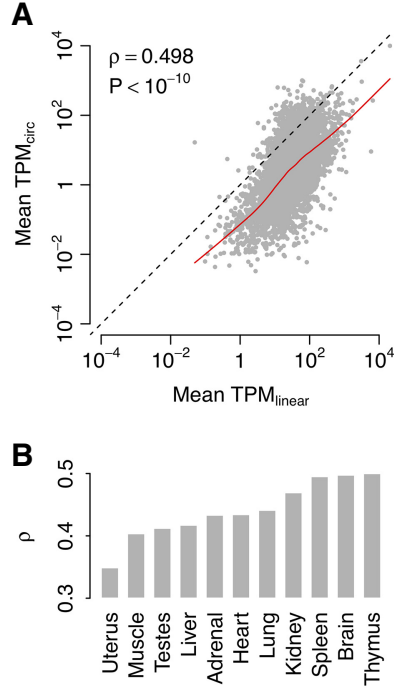
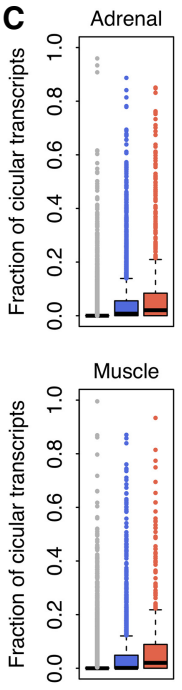
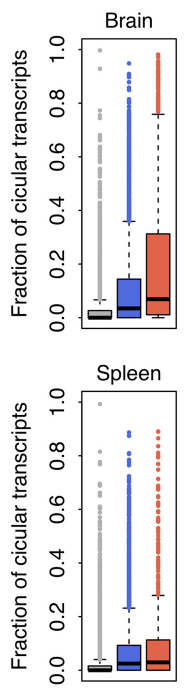
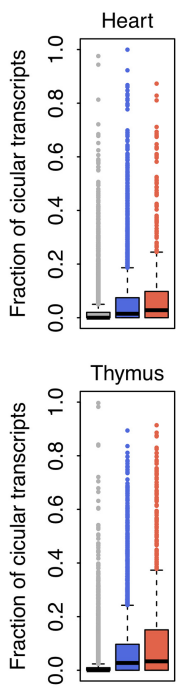
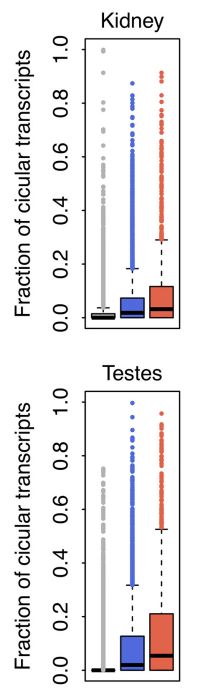
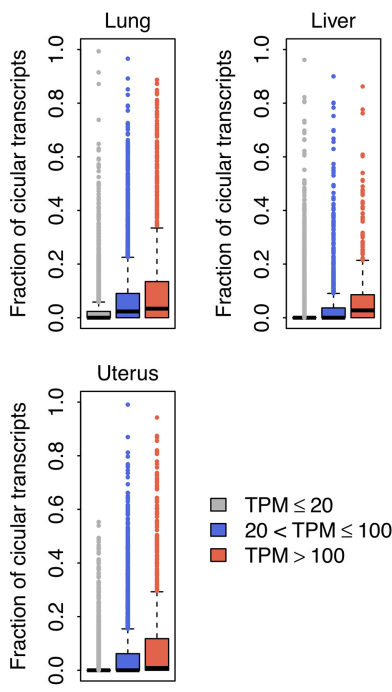

ㄴ.

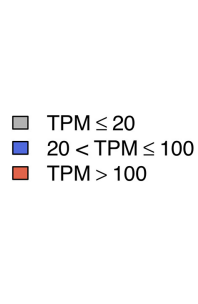

FIGURE 2. Relationship between the expression of circRNAs and their linear counterparts. (A) Correlation between mean TPM linear and mean $T P M_{\text {circ }}$ values in the rat brain. Each dot represents one host gene. The dots above the diagonal denote the host genes with higher circRNA expression relative to their linear transcripts in the brain. The red curve represents Lowess smoothed data. The correlation coefficient ( $\rho$ ) and $P$-value were calculated with Spearman's rank correlation test. (B) Comparison of the correlation coefficient $(\rho)$ calculated between mean TPM linear $_{\text {and }}$ mean TPM circ values across the 11 rat tissues by Spearman's rank correlation test. (C) Comparison of the fraction of circular transcripts for genes categorized by expression level. 

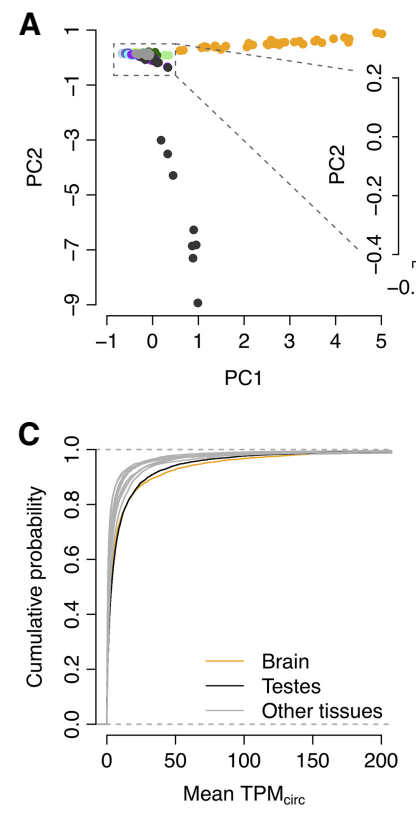

D

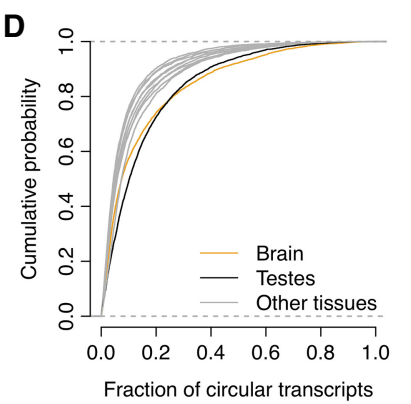

B

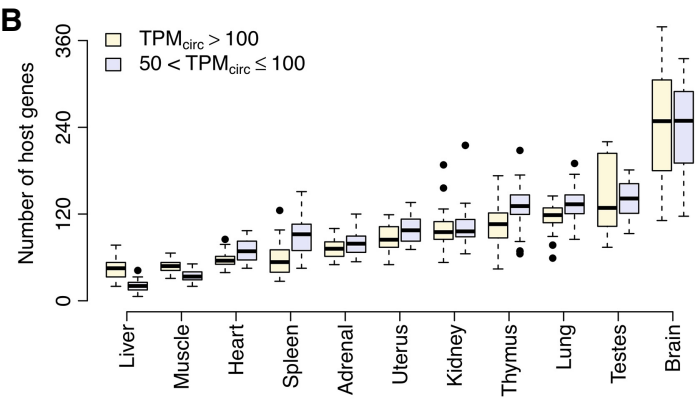

E

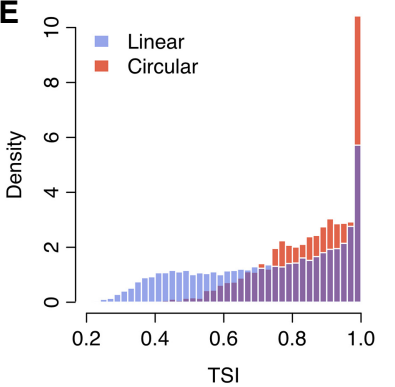

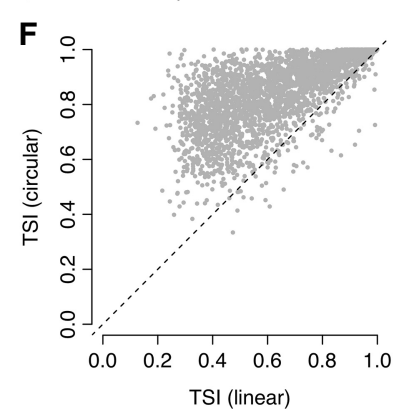

FIGURE 3. Tissue specificity of circRNA expression. (A) PCA of circRNA expression. Each dot represents one tissue sample. (PC1) First principal component, (PC2) second principal component. (B) Number of host genes with high circRNA expression. Genes with relatively higher TPM circ were categorized into two groups: $T P M_{\text {circ }}>100$ and $50<T P M_{\text {circ }}<100$. Each boxplot consists of 32 samples from the specific tissue type, except for the testes and uterus, for which only 16 samples are included. (C) Cumulative distribution of the mean TPM circ values across all 11 tissue types. (D) Cumulative distribution of the fraction of circular transcripts across all 11 tissue types. (E) Histogram of the TSI of both linear and circular transcripts. (F) Paired comparison of TSI between linear and circular transcripts. Each dot represents one host gene. The dots above the diagonal denote the host genes with a higher circRNA TSI than their linear counterparts.

samples. We found that the samples from the same tissue type tended to cluster together according to the first and second principal components (Fig. 3A), which suggests that circRNAs are expressed in a tissue-specific manner. Notably, the brain and testes samples showed extremely unique PCA patterns compared with the other tissues (Fig. 3A), which was further confirmed by the observation that the brain and testes exhibited the largest number of highly expressed circRNAs (Fig. 3B). Additionally, the mean TPM circ values and the fraction of circular transcripts were significantly higher in the brain and testes than in the other tissue types (Kolmogorov-Smirnov test: $P<10^{-5}$; Fig. 3C,D).

To compare tissue specificity between circRNAs and mRNAs, we calculated the tissue specificity index (TSI) for both the circular and linear transcripts of each host gene (see Materials and Methods for details). A higher TSI indicates higher tissue specificity. We found that there were more circRNAs than linear RNAs showing a TSI>0.8 (Fig. 3E). Paired comparisons indicated that the TSI of circular transcripts was significantly higher than the TSI of their corresponding linear counterparts (paired Wilcoxon test: $P<10^{-10}$; Fig. 3F). TSI values based on the fraction of circular transcripts showed the same trend when compared to their corresponding linear mRNAs (Supplemental Fig. S4), which suggests that the higher tissue specificity of circular transcripts is independent of the expression level of their host genes. All these results suggest that, although mRNAs exhibit a tissue-specific expression pattern (Supplemental Fig. S5), circRNAs are expressed in a more dynamic manner among different rat tissues and show higher tissue specificity.

\section{A map of tissue-specific circRNAs and their potential physiological functions}

To understand whether the observed tissue-specific circRNA expression is relevant to the physiological function of the specific tissue, we performed a hierarchical clustering analysis based on the dynamic expression of the tissue-specific circRNAs across 320 rat tissue samples. We observed that the samples from the same tissue type were clustered into a single group, and each tissue type exhibited one or more unique tissue-specific circRNA block(s) (Fig. 4A). Tissues with similar physiological functions tended to group together and share common circRNA blocks, as observed for the thymus and spleen, which play vital roles in the immune system (Fig. 4A). Additionally, the samples from two reproduction-related tissues, testes and uterus, were aggregated into one group as well (Fig. 4A). In contrast, the brain samples showed patterns distinct from those of other tissues (Fig. 4A).

To explore the potential functions of circRNAs in different tissues, we next examined the GOBP (Ashburner et al. 
A

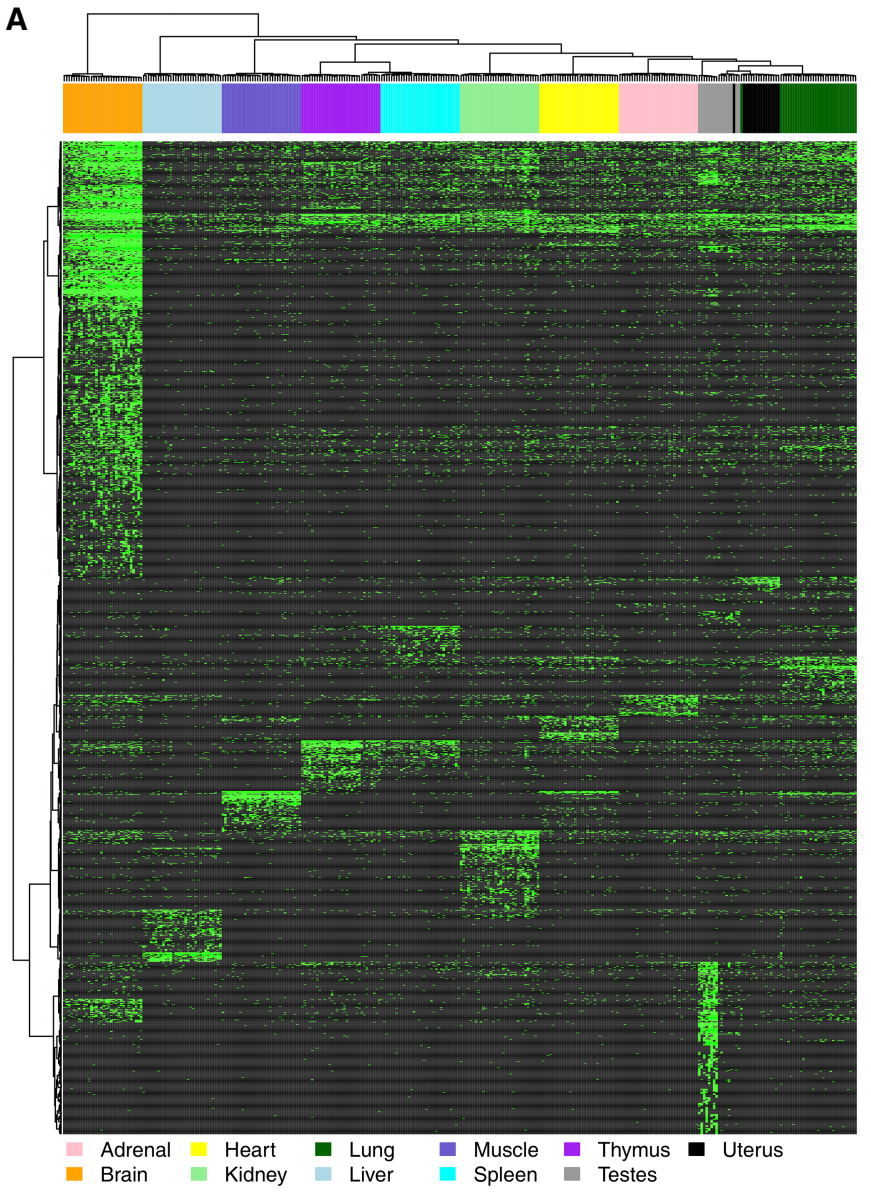

C
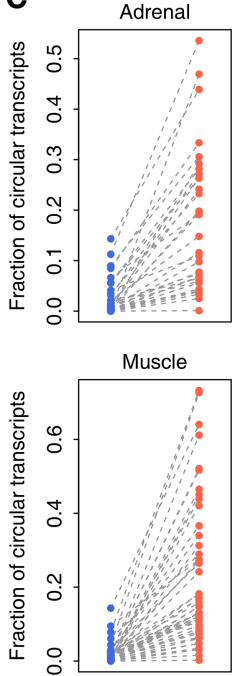
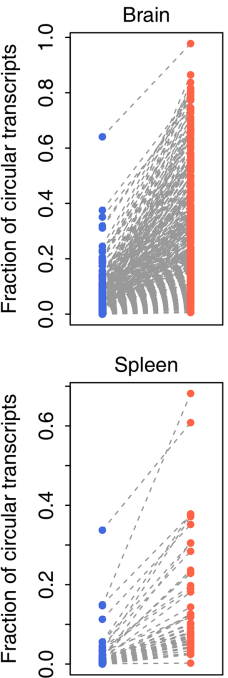
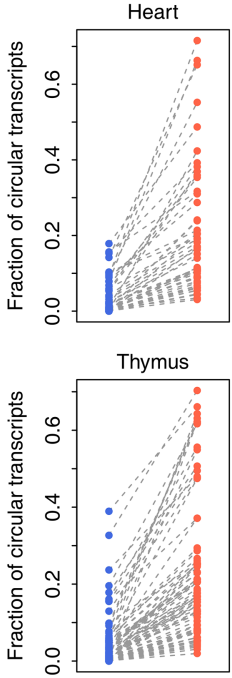

B

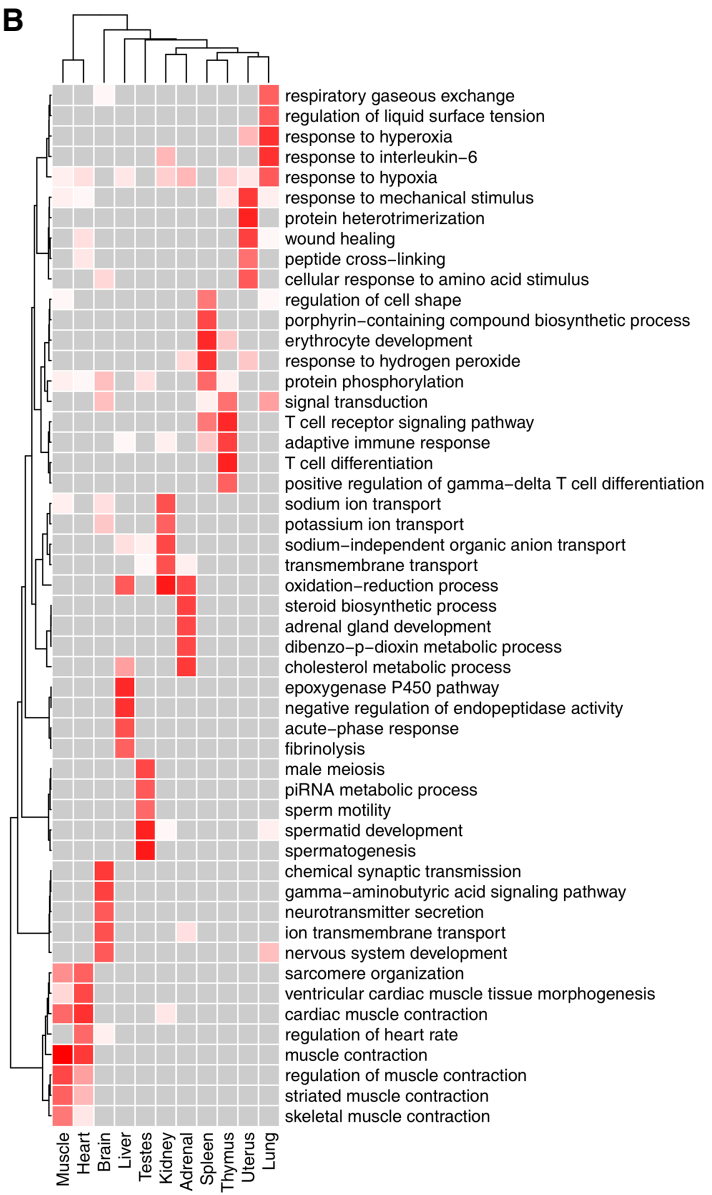

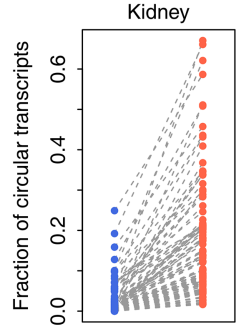

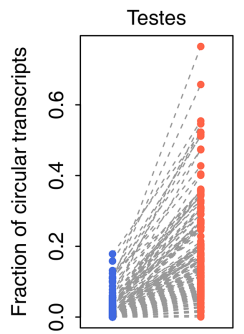

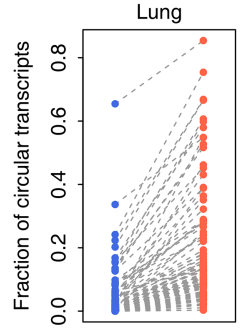
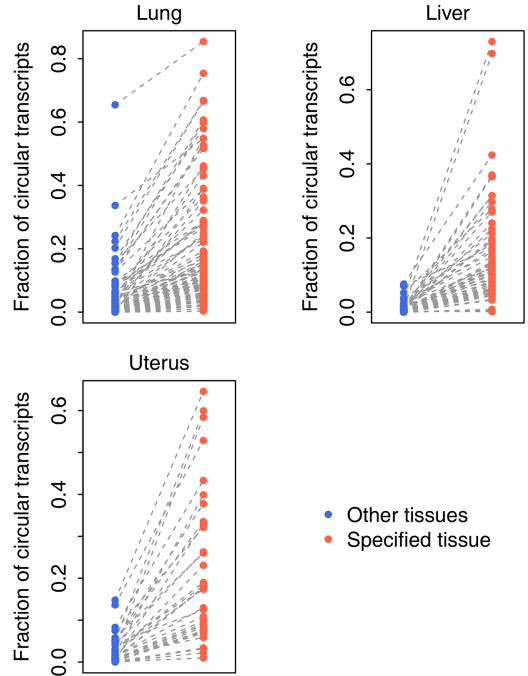

- Other tissues

- Specified tissue

FIGURE 4. Map of tissue-specific circRNAs. (A) Hierarchical clustering of tissue-specific circRNAs. Each green dot represents one expressed tissue-specific circRNA. (B) The top GOBP terms associated with the host genes of the tissue-specific circRNAs. For each tissue type, the top five GOBP terms are listed. The association between the GOBP terms and tissue-specific circRNAs was measured based on the Z-score calculated from Fisher's exact test. Darker red indicates a stronger association, while lighter red indicates a weaker association. Gray indicates no association. (C) Fraction of circular transcripts of tissue-specific circRNAs. Each dot represents one tissue-specific circRNA.

2000) terms associated with the host genes of the tissuespecific circRNAs. We found that the enriched GOBP terms were highly related to the biological/physiological function of each specific tissue (Fig. 4B). For example, the lung-specific circRNAs were associated with "respiratory gaseous exchange," "response to hyperoxia," 

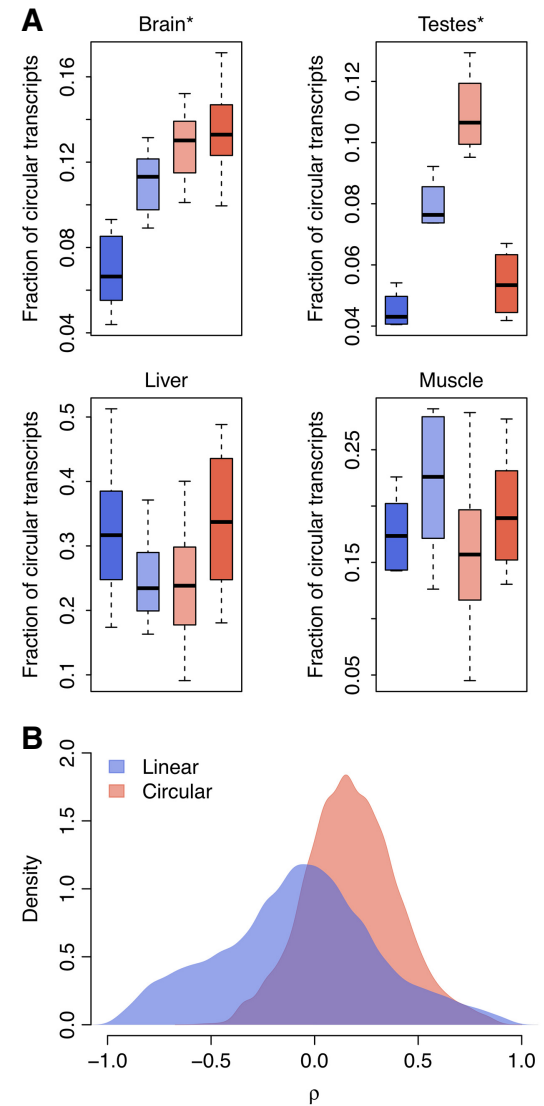
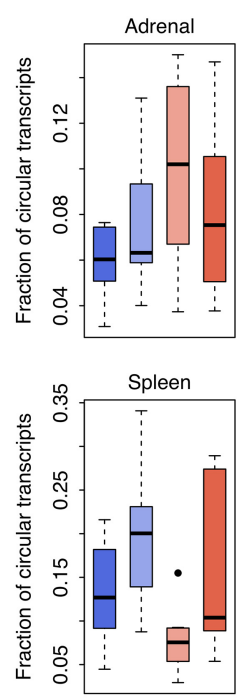

C

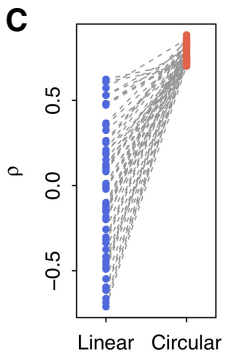

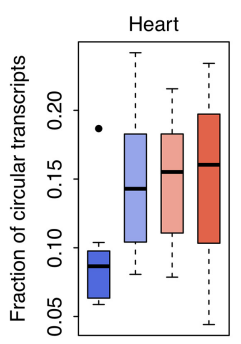
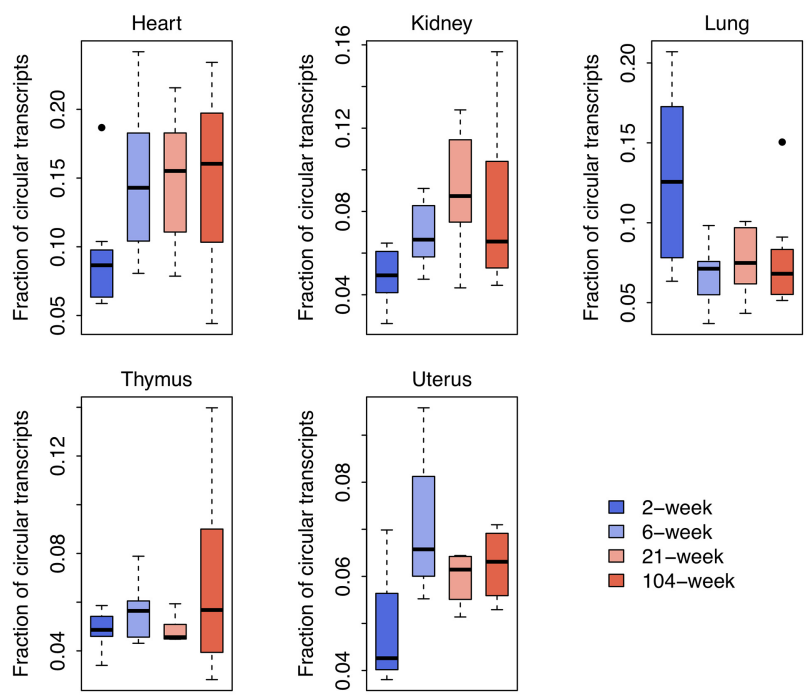

104-week

D

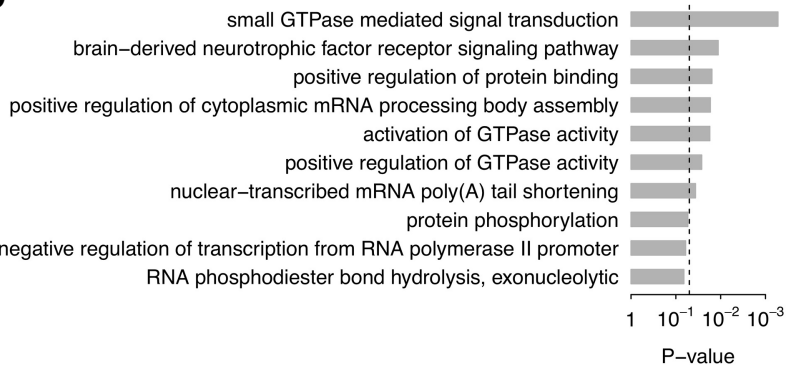

FIGURE 5. Age-dependent circRNA expression. (A) Relationship between age and overall circRNA abundance across the 11 tissue types. (B) Distribution of the correlation coefficients $(\rho)$ between age and the expression of linear/circular transcripts in the brain. The $\rho$ values were calculated with Spearman's rank correlation test. (C) Paired comparisons of $\rho$ between the circRNAs that accumulated with age $(\rho>0.7)$ and their linear counterparts in the brain. (D) Top 10 GOBP terms associated with the circRNAs that accumulated with age in the brain. The P-values were calculated with Fisher's exact test. The vertical dashed line indicates the significance level of $\alpha=0.05$.

and "response to hypoxia"; the brain-specific circRNAs were associated with "chemical synaptic transmission," "neurotransmitter secretion," and "nervous system development"; and the testes-specific circRNAs were associated with "sperm motility," "spermatid development," and "spermatogenesis" (Fig. 4B). All these results suggest that the tissue-specific expression pattern of circRNAs is important for all types of tissues to perform their biologi$\mathrm{cal} /$ physiological roles.

To gain further insight into the potential driving force causing the tissue-specific expression of circRNAs, we compared the splicing ratio of tissue-specific circRNAs in the specific tissue against that in all other tissue types. We observed a consistently higher fraction of circular transcripts for the tissue-specific circRNAs in an individual tissue (paired Wilcoxon test: $P<10^{-10}$; Fig. 4C). However, we observed an opposite pattern when tissue-specific mRNAs were considered (Supplemental Fig. S6). These results suggest that tissue-specific circRNAs may be positively regulated at the splicing or post-transcriptional level to ensure the proper function of the specific tissue.

\section{Age-dependent circRNA expression in the rat brain}

We next investigated the temporal changes in circRNA expression in rat tissues at four different developmental stages $(2,6,21$, and $104 \mathrm{wk})$. Although the overall fraction of circular transcripts was not longitudinally correlated with age for most of the tissues (Fig. 5A), the abundance of brain circRNAs was found to monotonically increase with age (Spearman's rank correlation test: $\rho=0.772$ and $P=2.3 \times 10^{-7}$; Fig. $5 \mathrm{~A}$ ), which is consistent with previous observations made in the brains of humans (Szabo et al. 2015), mice (You et al. 2015; Gruner et al. 2016), and flies (Westholm et al. 2014). We also investigated the age-dependent expression of each gene in the brain. The correlation coefficient $(\rho)$ between expression and age was calculated for both circular and linear transcripts. We found that the $\rho$ values of the circRNAs were significantly more positive than those of the linear RNAs ( $t$-test: $P<10^{-10}$; Fig. 5B). In particular, when we focused on the circRNAs with a strong positive correlation $(\rho>0.7$; Supplemental Table S1), we found that the $\rho$ values of their 
corresponding linear counterparts were significantly lower (paired t-test: $P<10^{-10}$; Fig. 5C). Even when we randomly generated circRNA sets $(n=1000)$ containing the same number of circRNAs listed in Supplemental Table S1, we found that, among $>95 \%$ of the resampled circRNA sets, the $\rho$ values of circRNAs were significantly higher than those of their corresponding linear counterparts (onetailed paired t-test: $P<0.05$; Supplemental Fig. S7). All these results suggest that the age-dependent expression of brain circRNAs is largely independent of linear transcripts and, at least to a certain extent, is not a secondary consequence of host gene expression. Gene ontology analysis indicated that the circRNAs that accumulated with age were enriched in GOBP terms linked to neural development, such as "brain-derived neurotrophic factor receptor signaling pathway" and "activation of GTPase activity" (Fig. 5D). Our data support the concept that circRNAs in the brain might play an essential role in regulating synaptic plasticity and neuronal differentiation (Hanan et al. 2017).

\section{Age-dependent circRNA expression in testes}

Interestingly, circRNA expression in rat testes showed a more dynamic pattern during the four developmental stages $(2,6,21$, and $104 \mathrm{wk})$ we examined (Fig. 5A). Unlike the monotonic increase of circRNA abundance in the rat brain, the overall fraction of circular transcripts in testes accumulated linearly for the first three developmental stages $(2,6$, and $21 \mathrm{wk})$, which nicely mirrored the stages approaching sexual maturity, during which the male reaches reproductive peak. However, the abundance of circRNAs drastically decreased at the age of $104 \mathrm{wk}$ (Fig. $5 A)$, at which stage the rats could be classified as aged males with declining reproduction. Moreover, by further examining the correlation between expression and developmental stage for individual genes, we showed that, for the circRNAs exhibiting monotonically increased expression from 2 wk to 21 wk ( $\rho>0.6$; Supplemental Table S2), the $\rho$ values of their corresponding linear counterparts were significantly lower (paired t-test: $P<10^{-10}$; Fig. 6A). Even for the randomly generated circRNA sets $(n=1000)$ of identical size (the same number of circRNAs included in the upper panel of Fig. 6A), the $\rho$ values of circRNAs were significantly higher than those of their corresponding linear counterparts in all the resampled circRNA sets (onetailed paired t-test: $P<0.05$; Supplemental Fig. S8). On the contrary, for the circRNAs down-regulated from 21 wk to 104 wk $(\rho<-0.6$; Supplemental Table S2), the $\rho$ values of their linear counterparts were significantly increased (paired t-test: $P<10^{-10}$; Fig. 6A). A resampling test indicates that, when generating random circRNA sets $(n=1000)$ of identical size (the same number of circRNAs included in the lower panel of Fig. 6A), the $\rho$ values of circRNAs were also significantly lower than those

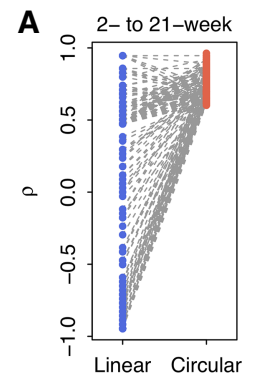

B
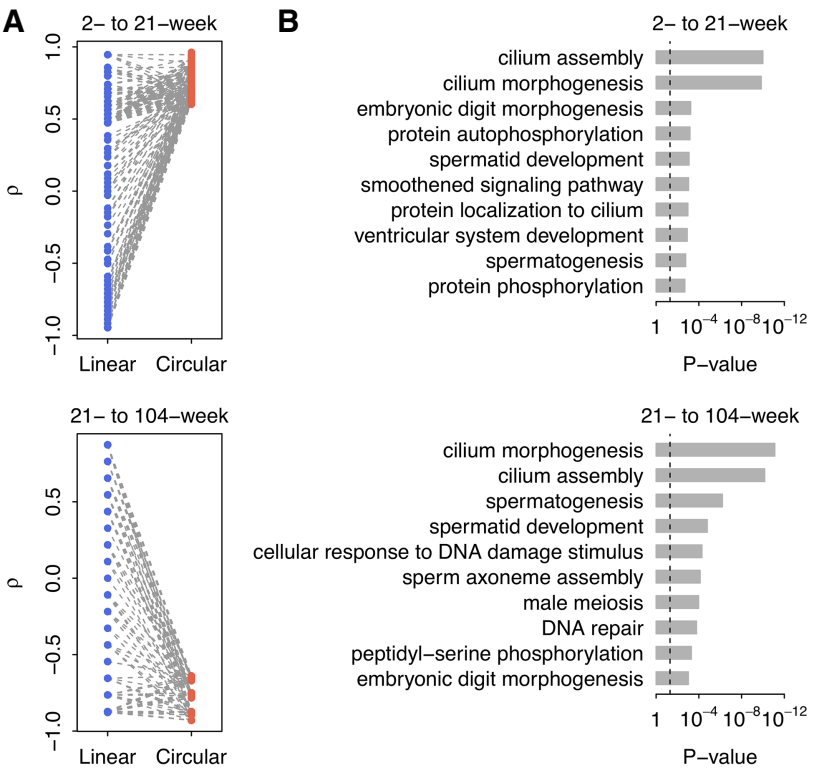

C
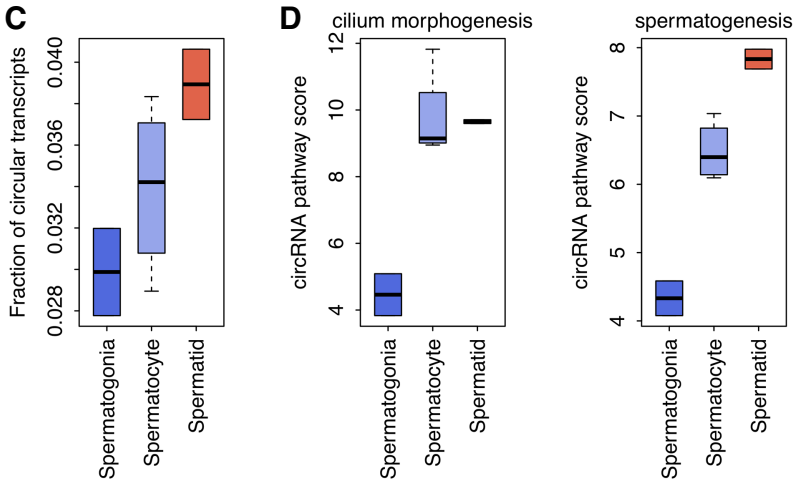

FIGURE 6. Age-dependent circRNAs in testes. (A) Paired comparisons of correlation coefficients $(\rho)$ between the age-dependent circRNAs $(\rho>0.6)$ and their linear counterparts in rat testes. The $\rho$ values were calculated between age and the expression of linear/circular transcripts using Spearman's rank correlation test. $(B)$ The top 10 GOBP terms associated with the age-dependent circRNAs in rat testes. The $P$-values were calculated with Fisher's exact test. The vertical dashed line denotes the significance level of $\alpha=0.05$. (C) The circRNA abundance in mouse spermatogenic cells was categorized by distinct stages (spermatogonia, spermatocytes, and spermatids, sequentially). (D) The circRNA expression-based pathway scores of the mouse spermatogenic cells. We calculated the circRNA pathway scores for both the "cilium morphogenesis" and "spermatogenesis" GOBP terms. Higher pathway scores indicate higher overall circRNA expression for the specific GOBP terms.

of their corresponding linear counterparts in all the resampled sets (one-tailed paired t-test: $P<0.05$; Supplemental Fig. S8). All these results suggest that the age-dependent expression of testes circRNAs is also somewhat independent of host gene expression and may represent a unique signature of the stage-specific reproductive performance of the male. Indeed, gene ontology analysis further revealed that both the genes up-regulated from 2 wk to 21 wk and the genes down-regulated from 21 wk to 104 wk 
were significantly enriched in GOBP terms linked to spermatogenesis, such as "cilium morphogenesis," "spermatid development," and "spermatogenesis" (Fig. 6B).

The age-specific pattern of circRNAs found in rat testes might be correlated at the molecular level with certain stages of sperm development that are indicative of specific functions. To test this hypothesis, we further examined the stage-specific circRNA expression profiles of different spermatogenic cells (spermatogonia, spermatocyte, and spermatid) from a published data set for mice (Lin et al. 2016) and cross-analyzed the spermatogenic stagespecific gene categories with the age-specific circRNA signatures observed in rats. Interestingly, we found that the overall fraction of circular transcripts increased monotonically with the spermatogenesis stage in mice (spermatogonia, spermatocytes, and spermatids, sequentially) (Spearman's rank correlation test: $\rho=0.772$ and $P=2.5 \times$ $10^{-2}$; Fig. 6C). We also found that the circRNA expression-based pathway scores (see Materials and Methods for details) for both the "cilium morphogenesis" and "spermatogenesis" GOBP terms (most dynamically associated with age in rat testes) were positively correlated with the spermatogenic stage in mice (Spearman's rank correlation test: $\rho=0.772$ and $P=2.5 \times 10^{-2}$ for "cilium morphogenesis," and $\rho=0.926$ and $P=9.6 \times 10^{-4}$ for "spermatogenesis"; Fig. 6D). These results further support our hypothesis that the circRNAs present in testes not only are highly associated with the developmental stages of spermatogenesis but also could be harnessed as a biomarker for reproductive aging.

\section{DISCUSSION}

In this study, we performed a thorough investigation of circRNA transcriptomes in the rat BodyMap data set. In comparison with existing studies (Guo et al. 2014; Conn et al. 2015; Rybak-Wolf et al. 2015; You et al. 2015), we systematically analyzed the expression profiles of both circular and linear transcripts in 320 rat samples across 11 tissue types and four developmental stages and particularly focused on the relationships and differences between the expression of circRNAs and mRNAs. Understanding the relationship between circRNAs and mRNAs is helpful for answering several open questions regarding circRNA biogenesis and function, including (i) whether circRNAs are simply by-products of mRNA transcription, (ii) whether circRNAs show expression patterns similar to those of mRNAs across different tissue types, and (iii) whether the association of circRNAs with aging is similar to that of mRNAs.

First, we asked whether circRNAs are simply by-products of the transcription and splicing of their host genes. If not, what are the factors affecting the final products of circular transcripts? Several groups (Liang and Wilusz 2014; Conn et al. 2015; Rybak-Wolf et al. 2015; You et al. 2015) have investigated the correlation between the expression levels of circRNAs and their corresponding mRNAs. Guo et al. (2014) and Salzman et al. (2013) found that the relative expression levels of circRNAs and their linear counterparts can differ between cell types and tissue types. Two other studies showed that the differential changes in many circRNAs are independent of the changes in the expression of their host linear transcripts upon neuronal differentiation (Rybak-Wolf et al. 2015; You et al. 2015). These results suggest that circRNAs are not simply by-products of occasional aberrant splicing (Chen 2016). The current study provides further novel observations regarding the correlation between circRNAs and mRNAs. We found a positive correlation between circRNA expression and the expression of cognate linear mRNAs in all tissue samples (Fig. 2A,B; Supplemental Fig. S2). The obvious explanation of this correlation is that circRNAs are controlled at the transcription level of their host genes since they are effective splice products. On the other hand, some circRNAs, such as exon-intron circRNAs, can enhance the expression of their parental genes in cis ( $\mathrm{Li}$ et al. 2015b). The cis regulation of circRNAs on its parental gene may have some additional effects on the relationship between circRNA and linear mRNA expression as well. Furthermore, our data support the concept that circRNAs are more sensitive to changes in host gene expression. Generally, increased host gene transcription levels tend to increase the expression of both mRNAs and circRNAs; thus, if there is no splicing regulation of circRNA expression, no substantial differences in the ratio of the expression of circular and linear transcripts should be observed. However, we observed a consistently higher fraction of circular transcripts among highly expressed genes in all tissues (Fig. 2C), which suggests that both transcriptional and post-transcriptional regulators contribute to the final output of circRNA expression. The correlation between the splicing efficiency of circRNAs and the total expression of their host genes may be explained by the competitive splicing between linear and circular transcripts (AshwalFluss et al. 2014). Interestingly, Rybak-Wolf et al. (2015) observed a negative correlation between the logarithm of gene expression and the circular to linear ratio, which is exactly opposite the trend we observed (Fig. 2C). Although the cause of these paradoxical observations is currently unknown, a recent study (Liang et al. 2017a) explored how the ratio of linear and circRNA is controlled and identified many core spliceosome and transcription termination factors that control the RNA outputs of reporter and endogenous genes. It has also been suggested that circRNAs become the preferred gene output when core spliceosome or transcription termination factors are depleted. In this context, it was reasonable to observe a positive correlation, rather than a negative correlation, between the ratio of circular transcripts to linear transcripts since the required core spliceosomes and transcription termination factors 
are likely to be insufficient for genes with higher expression levels. Technically, the computational pipeline used in Rybak-Wolf et al. (2015) is unable to distinguish the sequencing reads sampled from the exonic region of circular transcripts and those derived from the same genomic region of canonical linear transcripts. This potentially underestimates the expression level of circRNAs and overestimates that of their cognate linear mRNAs. In our analysis, we used our newly developed model-based pipeline, Sailfish-cir (Li et al. 2017), to simultaneously quantify the expression of both circRNAs and mRNAs from RNA-seq data in a more precise manner (Gao and Zhao 2018). Taken together, our observations not only confirm the importance of competitive splicing against linear transcripts in circRNA production but also imply the significance of host gene transcription in determining the final output of the corresponding spliced circRNA.

Second, we investigated the relevance of both circRNAs and mRNAs for tissue-specific phenotypes. Consistent with previous studies (Salzman et al. 2013; Ashwal-Fluss et al. 2014; Conn et al. 2015; Rybak-Wolf et al. 2015; Starke et al. 2015; Szabo et al. 2015; You et al. 2015), we observed that both circRNAs (Figs. 3A, 4A) and mRNAs (Supplemental Fig. S5) exhibited specific expression profiles across different tissues. Our data further highlighted that the expression of these tissue-specific circRNAs was closely related to the physiological functions of the specific tissue (Fig. 4B). It is widely accepted that the tissue-specific expression of linear mRNAs is related to the function of multicellular tissues and human diseases (Dezso et al. 2008; Greene et al. 2015; The GTEx Consortium 2015; Melé et al. 2015). Therefore, it is interesting to explore the differences between circRNAs and mRNAs in terms of tissue specificity and their contribution to tissue phenotypes. In our study, we observed that the tissue specificity of circRNAs was consistently higher than that of linear mRNAs (Fig. 3E,F). However, care should be taken on this conclusion, because the rat circRNA repertoire in our study may be underestimated given the limited number of samples of each tissue type and the relatively low expression level of circRNAs in the rRNA-depleted RNAseq libraries. Additionally, a higher splicing ratio was observed for tissue-specific circRNAs (Fig. 4C), but not for tissue-specific linear RNAs (Supplemental Fig. S6), which suggests that the contribution of circRNAs to tissue specificity is somewhat independent of their cognate linear mRNAs. This finding is in accordance with the previous finding that the changes in circRNAs upon neuronal differentiation are independent of the changes of their linear counterparts (Rybak-Wolf et al. 2015; You et al. 2015; Gruner et al. 2016). Taken together with the evidence presented in previous studies (Salzman et al. 2013; AshwalFluss et al. 2014; Conn et al. 2015; Rybak-Wolf et al. 2015; Starke et al. 2015; Szabo et al. 2015; You et al. 2015), we propose that while both forms of RNA transcripts can make independent contributions to tissue-specific functions, circRNAs may be more relevant to tissue specificity than linear mRNAs.

Third, we explored the accumulation of circRNAs and linear mRNAs across developmental stages in different tissues. Previous studies have shown that circRNAs gradually accumulate with age in brain samples, which has been observed in several organisms, such as humans (Szabo et al. 2015), mice (You et al. 2015; Gruner et al. 2016), and flies (Westholm et al. 2014). We confirmed a monotonic increase of circRNAs in the rat brain samples (Fig. 5A), while most other tissues, such as the heart, liver, and lungs, did not present a consistent association of circRNAs with age (Fig. 5A; Supplemental Table S3), which is consistent with the observations made by Gruner et al. (2016). They (Gruner et al. 2016) investigated the changes of circRNAs in mouse heart samples and found no significant changes in circRNA expression between young and aged mice.

In addition to the age-dependent circRNA profile found in the brain, another major novel discovery of our study is a previously unidentified, dynamic age-dependent pattern in the testes, where circRNA levels mirror sexual maturity and the robustness of male reproduction. Our further pathway-level analysis revealed that the circRNA populations showing age-sensitive changes are essential for spermatogenesis, which was cross-analyzed and confirmed in mouse developmental stage-specific spermatogenetic cells. In particular, the potential function of circRNAs in cilium morphogenesis (essential for the formation of the spermatid flagellum) is very interesting, which may spur future extensive basic and translational studies.

Finally, we surveyed the potential causes of the age-dependent circRNA expression observed in the brain and testes. One obvious cause is the much lower degradation rate of circRNAs than that of mRNAs (Enuka et al. 2016), which explains the enrichment of circRNAs in exosomes (Li et al. 2015a) and several anucleate blood components, such as platelets and red blood cells (Alhasan et al. 2016). Given that neurons show relatively low proliferation, circRNAs could accumulate in neurons during aging (Gruner et al. 2016). However, increased circRNA stability cannot explain the circRNA accumulation observed in several other tissues. For example, although cardiomyocytes show similar proliferation rates to neurons, heart circRNAs do not continuously accumulate during aging (Fig. 5A). Therefore, factors other than circRNA stability itself should account for circRNA accumulation during aging. For example, Harries et al. (2011) observed that two splicing factors, SRFR6 and SRFS1, were down-regulated in human aging. The decreased expression of splicing factors with age may be related to the accumulation of circRNAs. Indeed, age-dependent circRNA expression has been implicated in several biological processes, including neuronal differentiation (Rybak-Wolf et al. 2015; You et al. 2015), epithelial-mesenchymal transition (Conn 
et al. 2015), and fetal development (Szabo et al. 2015). Therefore, it is reasonable to assume that the accumulation of circRNAs in the brain and testes is a regulatory outcome on circRNA expression to ensure proper physiological functions at different developmental stages.

In conclusion, we present a comprehensive view of circRNA expression profiles and their relevance to linear mRNAs across different rat tissues and developmental stages. We propose that circRNAs have important functional implications for tissue phenotypes and development, which are independent of their linear counterparts. Highlights of our study include the findings that the testis is the most sensitive organ showing age-dependent changes in circRNA levels and that circRNAs could be essential in regulating the process of spermatogenesis and might be used as a biomarker of reproductive maturity and aging. Nevertheless, we need to point out that, though we mainly focus on the cis-effect of circRNAs, existing studies have suggested that circRNAs can also play trans-regulatory roles as miRNA (Hansen et al. 2013) and/or RNA binding protein sponges (Conn et al. 2015). In future work, it will be very interesting to explore the trans-effect of circRNAs on rat tissue phenotypes and development.

\section{MATERIALS AND METHODS}

\section{Raw RNA-seq data}

To explore the dynamic expression of both circular and linear transcripts based on a single data set, we downloaded the raw RNA-seq data of the rat BodyMap data set from the NCBI GEO database (Barrett et al. 2013) under accession code GSE53960. In the rat BodyMap data set, Yu et al. $(2014 a, b)$ constructed and sequenced 320 rRNA-depleted RNA-seq libraries containing samples from 11 rat tissue types (adrenal gland, brain, heart, kidney, liver, lung, muscle, spleen, thymus, testes [male only], and uterus [female only]) from both sexes of Fischer 344 rats across four developmental stages $(2,6,21$, and 104 wk). For each developmental stage, four male and four female biological replicates were obtained from adrenal, brain, heart, kidney, liver, lung, muscle, spleen, and thymus tissues (Supplemental Table S4). For the testes/uterus, four male/female replicates were included for each developmental stage (Supplemental Table S4). To further investigate age-sensitive testicular circRNA expression, we also obtained raw RNA-seq data for mouse spermatogenic cells from the NCBI GEO database (Barrett et al. 2013) under accession code GSE75826. This data set contains rRNA-depleted RNA-seq data from three different types of spermatogenic cells: spermatogonia, spermatocytes, and spermatids (Lin et al. 2016).

\section{Identification and quantification of circRNAs}

For each sample in the rat BodyMap data set (Yu et al. 2014a,b), we filtered the raw RNA-seq reads by removing adaptor sequences, contamination, and low-quality reads and assessed data qual- ity using RNA-SeQC (DeLuca et al. 2012). Next, we identified all circRNAs in the sample using CIRI (Gao et al. 2015) with default parameter settings. We used the mRNA exon structures in Ensembl rat gene annotation (Cunningham et al. 2015) (release 91) as the reference to determine the exons within circRNAs. To reduce potential false positives of identified circRNAs, we constructed a rat circRNA repertoire by retaining all circRNAs with at least one back-splicing read in at least two samples of the same tissue type. After circRNA identification, we quantified the expression levels of all identified circular transcripts and known linear transcripts in the Ensembl rat gene annotation (Cunningham et al. 2015) (release 91) using Sailfish-cir (Li et al. 2017) with default settings. For each host gene, we calculated the TPM values of both circular and linear transcripts. The same computational pipeline was used to identify and quantify mouse circRNAs from mouse spermatogenic cells.

\section{Evolutionary analysis of rat circRNAs}

We performed the evolutionary analysis of rat circRNAs at two levels. We compared the conservation levels of nucleotide sequences in the coding region of exonic circRNAs against those of linear mRNAs. For each exonic circRNA, we used the transcript structure of the longest linear mRNA transcript of its host gene. We downloaded the PhastCons scores for the rat genome from the UCSC Genome Browser database (Casper et al. 2018). The base-by-base PhastCons score represents the posterior probability that the corresponding alignment column is generated by the conserved state (Siepel et al. 2005). While a PhastCons score close to zero means that the site is evolutionary neutral, a PhastCons score close to one implies that the site is evolutionarily conserved. For each exonic circRNA, we extracted the PhastCons scores at all coding positions in its host gene and calculated the average PhastCons scores at all three codon positions for exons in circular transcripts and those exclusively in linear transcripts. In addition to nucleotide conservation levels, we analyzed the evolutionary gains and losses of rat circRNAs in the mammalian lineage. To this end, we downloaded the human circRNA repertoire from circBase (Glažar et al. 2014) and the mouse circRNA repertoire from circNet (Liu et al. 2016). One-to-one gene ortholog tables between humans, mice, and rats were downloaded from Ensembl (Vilella et al. 2009). CircRNAs that originated from orthologous genomic regions among different species were defined as orthologous circRNAs. We classified all the rat circRNAs with human and mouse orthologs as mammaliancommon circRNAs. The rat circRNAs with mouse orthologs but without human orthologs were defined as rodent-specific circRNAs. The rat circRNAs without either human or mouse orthologs were deemed rat specific.

\section{Tissue specificity}

To evaluate the variability of both circRNA and mRNA expression, we calculated the tissue specificity of each host gene. The TSI "tau" method developed by Yanai et al. (2005) was applied here as follows:

$$
T S I=\sum_{i=1}^{N}\left(1-r_{i}\right) /(N-1)
$$


Here, TSI is the tissue specificity index; $N$ is the number of tissue types; and $r_{i}$ is the mean expression in tissue $i$ normalized to the maximum mean expression in any tissue. The TSI value ranges from zero to one, and a higher TSI implies higher tissue specificity.

\section{CircRNA expression-based pathway score}

The FAIME algorithm (Yang et al. 2012) was applied to assign circRNA expression-based pathway scores for both the "cilium morphogenesis" and "spermatogenesis" GOBP terms for mouse spermatogenic cells. The FAIME method computes gene-set scores using the rank-weighted gene expression of individual samples, which converts each sample's transcriptomic information to molecular mechanisms (Yang et al. 2012). A higher circRNA expression-based pathway score indicates an overall increase in the abundance of the circRNAs within the given GOBP term.

\section{Statistical analyses}

All the statistical analyses were conducted on the R platform. PCA was used in this study to visualize the tissue specificity of circRNA expression and was performed with the "dudi.pca" function in the "ade4" library. Hierarchical clustering was conducted to visualize tissue-specific circRNAs. The corresponding heatmap was generated using the "heatmap.2" function in the "gplots" library with Ward's method. The t-test, Wilcoxon test, Spearman's rank correlation test, Kolmogorov-Smirnov test, and Fisher's exact test applied in this study were performed with the "t.test", "wilcox.test", "cor.test", "ks.test", and "fisher.test" functions, respectively.

\section{SUPPLEMENTAL MATERIAL}

Supplemental material is available for this article.

\section{ACKNOWLEDGMENTS}

We thank the members of the SEQC consortium for making the rat BodyMap RNA-seq data set freely accessible. This work was supported in part by the National Natural Science Foundation of China (61372164, 61471112, and 61571109), the Key Research and Development Program of Jiangsu Province (BE2016002-3), and the Fundamental Research Funds for the Central Universities (2242017K3DN04). This work was also supported by start-up grants provided by the University of Nevada to T.Z. The authors confirm that the funders had no influence over the study design, content of the article, or selection of this journal.

Received May 1, 2018; accepted August 3, 2018.

\section{REFERENCES}

Alhasan AA, Izuogu OG, Al-Balool HH, Steyn JS, Evans A, Colzani M, Ghevaert C, Mountford JC, Marenah L, Elliott DJ, et al. 2016. Circular RNA enrichment in platelets is a signature of transcriptome degradation. Blood 127: e1-e11.

Andergassen D, Dotter CP, Wenzel D, Sigl V, Bammer PC, Muckenhuber M, Mayer D, Kulinski TM, Theussl HC,
Penninger JM, et al. 2017. Mapping the mouse Allelome reveals tissue-specific regulation of allelic expression. Elife 6: e25125.

Ashburner M, Ball CA, Blake JA, Botstein D, Butler H, Cherry JM, Davis AP, Dolinski K, Dwight SS, Eppig JT, et al. 2000. Gene ontology: tool for the unification of biology. The Gene Ontology Consortium. Nat Genet 25: 25-29.

Ashwal-Fluss R, Meyer M, Pamudurti NR, Ivanov A, Bartok O, Hanan M, Evantal N, Memczak S, Rajewsky N, Kadener S. 2014. circRNA biogenesis competes with pre-mRNA splicing. Mol Cell 56: 55-66.

Barrett $T$, Wilhite SE, Ledoux $P$, Evangelista C, Kim IF, Tomashevsky M, Marshall KA, Phillippy KH, Sherman PM, Holko M, et al. 2013. NCBI GEO: archive for functional genomics data sets-update. Nucleic Acids Res 41: D991-D995.

Barrett SP, Wang PL, Salzman J. 2015. Circular RNA biogenesis can proceed through an exon-containing lariat precursor. eLife 4: e07540.

Bonizzato A, Gaffo E, te Kronnie G, Bortoluzzi S. 2016. CircRNAs in hematopoiesis and hematological malignancies. Blood Cancer J 6: e483.

Casper J, Zweig AS, Villarreal C, Tyner C, Speir ML, Rosenbloom KR, Raney BJ, Lee CM, Lee BT, Karolchik D, et al. 2018. The UCSC Genome Browser database: 2018 update. Nucleic Acids Res 46: D762-D769.

Chen L-L. 2016. The biogenesis and emerging roles of circular RNAs. Nat Rev Mol Cell Biol 17: 205-211.

Chen Y, Li C, Tan C, Liu X. 2016. Circular RNAs: a new frontier in the study of human diseases. J Med Genet 53: 359-365.

Conn SJ, Pillman KA, Toubia J, Conn VM, Salmanidis M, Phillips CA, Roslan S, Schreiber AW, Gregory PA, Goodall GJ. 2015. The RNA binding protein quaking regulates formation of circRNAs. Cell 160: 1125-1134.

Conn VM, Hugouvieux V, Nayak A, Conos SA, Capovilla G, Cildir G, Jourdain A, Tergaonkar V, Schmid M, Zubieta C, et al. 2017. A circRNA from SEPALLATA3 regulates splicing of its cognate mRNA through R-loop formation. Nat Plants 3: 17053.

Cunningham F, Amode MR, Barrell D, Beal K, Billis K, Brent $S$, Carvalho-Silva D, Clapham P, Coates G, Fitzgerald S, et al. 2015. Ensembl 2015. Nucleic Acids Res 43: D662-D669.

DeLuca DS, Levin JZ, Sivachenko A, Fennell T, Nazaire M-DD, Williams C, Reich M, Winckler W, Getz G. 2012. RNA-SeQC: RNA-seq metrics for quality control and process optimization. Bioinformatics (Oxford, England) 28: 1530-1532.

Dezso Z, Nikolsky Y, Sviridov E, Shi W, Serebriyskaya T, Dosymbekov D, Bugrim A, Rakhmatulin E, Brennan RJ, Guryanov A, et al. 2008. A comprehensive functional analysis of tissue specificity of human gene expression. BMC Biol 6: 49.

Enuka Y, Lauriola M, Feldman ME, Sas-Chen A, Ulitsky I, Yarden Y. 2016. Circular RNAs are long-lived and display only minimal early alterations in response to a growth factor. Nucleic Acids Res 44: 1370-1383.

Fagerberg L, Hallström BM, Oksvold P, Kampf C, Djureinovic D, Odeberg J, Habuka M, Tahmasebpoor S, Danielsson A, Edlund K, et al. 2014. Analysis of the human tissue-specific expression by genome-wide integration of transcriptomics and antibodybased proteomics. Mol Cell Proteomics 13: 397-406.

Gao Y, Zhao F. 2018. Computational strategies for exploring circular RNAs. Trends Genet 34: 389-400.

Gao Y, Wang J, Zhao F. 2015. CIRI: an efficient and unbiased algorithm for de novo circular RNA identification. Genome Biol 16: 4.

Gao Y, Wang J, Zheng Y, Zhang J, Chen S, Zhao F. 2016. Comprehensive identification of internal structure and alternative splicing events in circular RNAs. Nat Commun 7: 12060.

Glažar P, Papavasileiou P, Rajewsky N. 2014. circBase: a database for circular RNAs. RNA 20: 1666-1670. 
Greene CS, Krishnan A, Wong AK, Ricciotti E, Zelaya RA, Himmelstein DS, Zhang R, Hartmann BM, Zaslavsky E, Sealfon SC, et al. 2015. Understanding multicellular function and disease with human tissue-specific networks. Nat Genet 47: 569-576.

Gruner H, Cortés-López M, Cooper DA, Bauer M, Miura P. 2016. CircRNA accumulation in the aging mouse brain. Sci Rep 6: 38907.

The GTEx Consortium. 2015. The Genotype-Tissue Expression (GTEx) pilot analysis: multitissue gene regulation in humans. Science 348: 648-660.

Guo JU, Agarwal V, Guo H, Bartel DP. 2014. Expanded identification and characterization of mammalian circular RNAs. Genome Biol 15: 409 .

Hanan M, Soreq H, Kadener S. 2017. CircRNAs in the brain. RNA Biol 14: 1028-1034.

Hansen TB, Jensen $\mathrm{Tl}$, Clausen BH, Bramsen JB, Finsen B, Damgaard CK, Kjems J. 2013. Natural RNA circles function as efficient microRNA sponges. Nature 495: 384-388.

Harries LW, Hernandez D, Henley W, Wood AR, Holly AC, BradleySmith RM, Yaghootkar H, Dutta A, Murray A, Frayling TM, et al. 2011. Human aging is characterized by focused changes in gene expression and deregulation of alternative splicing. Aging Cell 10: 868-878.

Jeck WR, Sharpless NE. 2014. Detecting and characterizing circular RNAs. Nat Biotechnol 32: 453-461.

Jeck WR, Sorrentino JA, Wang K, Slevin MK, Burd CE, Liu J, Marzluff WF, Sharpless NE. 2013. Circular RNAs are abundant, conserved, and associated with ALU repeats. RNA 19: 141-157.

Kanitz A, Gypas F, Gruber A, Gruber A, Martin G, Zavolan M. 2015. Comparative assessment of methods for the computational inference of transcript isoform abundance from RNA-seq data. Genome Biol 16: 150.

Kumar L, Shamsuzzama, Haque R, Baghel T, Nazir A. 2017. Circular RNAs: the emerging class of non-coding RNAs and their potential role in human neurodegenerative diseases. Mol Neurobiol 54: 7224-7234.

Leek JT, Scharpf RB, Bravo HC, Simcha D, Langmead B, Johnson WE, Geman D, Baggerly K, Irizarry RA. 2010. Tackling the widespread and critical impact of batch effects in high-throughput data. Nat Rev Genet 11: 733-739.

Legnini I, Di Timoteo G, Rossi F, Morlando M, Briganti F, Sthandier O, Fatica A, Santini T, Andronache A, Wade M, et al. 2017. CircZNF609 is a circular RNA that can be translated and functions in myogenesis. Mol Cell 66: 22-37 e29.

Li Y, Zheng Q, Bao C, Li S, Guo W, Zhao J, Chen D, Gu J, He X, Huang S. 2015a. Circular RNA is enriched and stable in exosomes: a promising biomarker for cancer diagnosis. Cell Res 25: 981-984.

Li Z, Huang C, Bao C, Chen L, Lin M, Wang X, Zhong G, Yu B, Hu W, Dai $L$, et al. 2015b. Exon-intron circular RNAs regulate transcription in the nucleus. Nat Struct Mol Biol 22: 256-264.

Li M, Xie X, Zhou J, Sheng M, Yin X, Ko EA, Zhou T, Gu W. 2017. Quantifying circular RNA expression from RNA-seq data using model-based framework. Bioinformatics 33: 2131-2139.

Liang D, Wilusz JE. 2014. Short intronic repeat sequences facilitate circular RNA production. Genes Dev 28: 2233-2247.

Liang D, Tatomer DC, Luo Z, Wu H, Yang L, Chen L-L, Cherry S, Wilusz JE. 2017a. The output of protein-coding genes shifts to circular RNAs when the pre-mRNA processing machinery is limiting. Mol Cell 68: 940-954.e3.

Liang G, Yang Y, Niu G, Tang Z, Li K. 2017b. Genome-wide profiling of Sus scrofa circular RNAs across nine organs and three developmental stages. DNA Res 24: 523-535.

Lin X, Han M, Cheng L, Chen J, Zhang Z, Shen T, Wang M, Wen B, $\mathrm{Ni}$ T, Han C. 2016. Expression dynamics, relationships, and transcriptional regulations of diverse transcripts in mouse spermatogenic cells. RNA Biol 13: 1011-1024.
Liu YC, Li JR, Sun CH, Andrews E, Chao RF, Lin FM, Weng SL, Hsu SD, Huang CC, Cheng C, et al. 2016. CircNet: a database of circular RNAs derived from transcriptome sequencing data. Nucleic Acids Res 44: D209-D215.

Melé M, Ferreira PG, Reverter F, DeLuca DS, Monlong J, Sammeth M, Young TR, Goldmann JM, Pervouchine DD, Sullivan TJ, et al. 2015. Human genomics. The human transcriptome across tissues and individuals. Science 348: 660-665.

Memczak S, Jens M, Elefsinioti A, Torti F, Krueger J, Rybak A, Maier L, Mackowiak SD, Gregersen LH, Munschauer M, et al. 2013. Circular RNAs are a large class of animal RNAs with regulatory potency. Nature 495: 333-338.

Memczak S, Papavasileiou P, Peters O, Rajewsky N. 2015. Identification and characterization of circular RNAs as a new class of putative biomarkers in human blood. PLoS One 10: e0141214.

Meng S, Zhou H, Feng Z, Xu Z, Tang Y, Li P, Wu M. 2017. CircRNA: functions and properties of a novel potential biomarker for cancer. Mol Cancer 16: 94.

Pamudurti NR, Bartok O, Jens M, Ashwal-Fluss R, Stottmeister C, Ruhe L, Hanan M, Wyler E, Perez-Hernandez D, Ramberger E, et al. 2017. Translation of circRNAs. Mol Cell 66: 9-21 e27.

Pollard KS, Hubisz MJ, Rosenbloom KR, Siepel A. 2010. Detection of nonneutral substitution rates on mammalian phylogenies. Genome Res 20: 110-121.

Qian Z, Liu H, Li M, Shi J, Li N, Zhang Y, Zhang X, Lv J, Xie X, Bai Y, et al. 2018. Potential diagnostic power of blood circular RNA expression in active pulmonary tuberculosis. EBioMedicine 27: 18-26.

Rybak-Wolf A, Stottmeister C, Glažar P, Jens M, Pino N, Giusti S, Hanan M, Behm M, Bartok O, Ashwal-Fluss R, et al. 2015. Circular RNAs in the mammalian brain are highly abundant, conserved, and dynamically expressed. Mol Cell 58: 1-16.

Salzman J, Chen RE, Olsen MN, Wang PL, Brown PO. 2013. Cell-type specific features of circular RNA expression. PLoS Genet 9: e1003777.

Siepel A, Bejerano G, Pedersen JS, Hinrichs AS, Hou M, Rosenbloom K, Clawson H, Spieth J, Hillier LW, Richards S, et al. 2005. Evolutionarily conserved elements in vertebrate, insect, worm, and yeast genomes. Genome Res 15: 1034-1050.

Starke S, Jost I, Rossbach O, Schneider T, Schreiner S, Hung L-H, Bindereif A. 2015. Exon circularization requires canonical splice signals. Cell Rep 10: 103-111.

Szabo L, Morey R, Palpant NJ, Wang PL, Afari N, Jiang C, Parast MM, Murry CE, Laurent LC, Salzman J. 2015. Statistically based splicing detection reveals neural enrichment and tissue-specific induction of circular RNA during human fetal development. Genome Biol 16: 126.

Veno MT, Hansen TB, Veno ST, Clausen BH, Grebing M, Finsen B, Holm IE, Kjems J. 2015. Spatio-temporal regulation of circular RNA expression during porcine embryonic brain development. Genome Biol 16: 245.

Vilella AJ, Severin J, Ureta-Vidal A, Heng L, Durbin R, Birney E. 2009. EnsemblCompara GeneTrees: complete, duplication-aware phylogenetic trees in vertebrates. Genome Res 19: 327-335.

Wang PL, Bao Y, Yee MC, Barrett SP, Hogan GJ, Olsen MN, Dinneny JR, Brown PO, Salzman J. 2014. Circular RNA is expressed across the eukaryotic tree of life. PLoS One 9: e90859.

Westholm JO, Miura P, Olson S, Shenker S, Joseph B, Sanfilippo P, Celniker SE, Graveley BR, Lai EC. 2014. Genome-wide analysis of Drosophila circular RNAs reveals their structural and sequence properties and age-dependent neural accumulation. Cell Rep 9: $1-15$.

Xia S, Feng J, Lei L, Hu J, Xia L, Wang J, Xiang Y, Liu L, Zhong S, Han L, et al. 2016. Comprehensive characterization of tissue-specific 


\section{Zhou et al.}

circular RNAs in the human and mouse genomes. Brief Bioinform 18: 984-992.

Yanai I, Benjamin H, Shmoish M, Chalifa-Caspi V, Shklar M, Ophir R, Bar-Even A, Horn-Saban S, Safran M, Domany E, et al. 2005. Genome-wide midrange transcription profiles reveal expression level relationships in human tissue specification. Bioinformatics 21: 650-659.

Yang X, Regan K, Huang Y, Zhang Q, Li J, Seiwert TY, Cohen EE, Xing HR, Lussier YA. 2012. Single sample expression-anchored mechanisms predict survival in head and neck cancer. PLoS Comput Biol 8: e1002350.

Yang Y, Fan X, Mao M, Song X, Wu P, Zhang Y, Jin Y, Yang Y, Chen L, Wang $Y$, et al. 2017. Extensive translation of circular RNAs driven by $N^{6}$-methyladenosine. Cell Res 27: 626-641.
You X, Vlatkovic I, Babic A, Will T, Epstein I, Tushev G, Akbalik G, Wang M, Glock C, Quedenau C, et al. 2015. Neural circular RNAs are derived from synaptic genes and regulated by development and plasticity. Nat Neurosci 18: 603-610.

Yu Y, Fuscoe JC, Zhao C, Guo C, Jia M, Qing T, Bannon DI, Lancashire L, Bao W, Du T, et al. 2014a. A rat RNA-seq transcriptomic BodyMap across 11 organs and 4 developmental stages. Nat Commun 5: 3230.

Yu Y, Zhao C, Su Z, Wang C, Fuscoe JC, Tong W, Shi L. 2014b. Comprehensive RNA-seq transcriptomic profiling across 11 organs, 4 ages, and 2 sexes of Fischer 344 rats. Sci Data 1: 140013.

Zhang X-O, Wang H-B, Zhang Y, Lu X, Chen L-L, Yang L. 2014. Complementary sequence-mediated exon circularization. Cell 159: 134-147. 

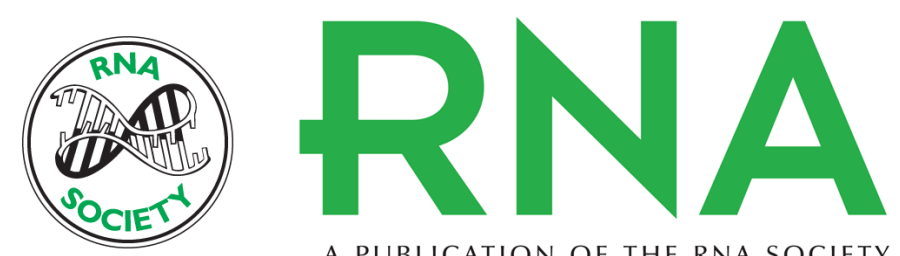

A PUBLICATION OF THE RNA SOCIETY

\section{Rat BodyMap transcriptomes reveal unique circular RNA features across tissue types and developmental stages}

Tong Zhou, Xueying Xie, Musheng Li, et al.

RNA 2018 24: 1443-1456 originally published online August 9, 2018

Access the most recent version at doi:10.1261/rna.067132.118

\section{Supplemental http://rnajournal.cshlp.org/content/suppl/2018/08/09/rna.067132.118.DC1 \\ Material}

References This article cites 66 articles, 11 of which can be accessed free at: http://rnajournal.cshlp.org/content/24/11/1443.full.html\#ref-list-1

Open Access Freely available online through the RNA Open Access option.

Creative This article, published in $R N A$, is available under a Creative Commons License Commons (Attribution 4.0 International), as described at

License http://creativecommons.org/licenses/by/4.0/.

Email Alerting Receive free email alerts when new articles cite this article - sign up in the box at the Service top right corner of the article or click here.

To subscribe to RNA go to:

http://rnajournal.cshlp.org/subscriptions 